\title{
Relation Between LV Diastolic Function and Aortic Compliance as Assessed by Transthoracic Echo
}

\author{
Inas Eweda, Sameh Samir, and Adham Abdeltawab*
}

Cardiology Department, Faculty of Medicine, Ain Shams University, Cairo, Egypt

\begin{abstract}
Arterial compliance and stiffness of the ascending aorta are important components of the elastic nature of the arterial system. Several pathological conditions are known to potentially affect arterial compliance properties as occurs with the arterial hypertension, and diabetes mellitus. Factors known to affect LV elasticity are similar to those affecting aortic elasticity.

Our aim in that study is to study correlation of aortic stiffness and LV diastolic function and the factors that affect each. We scanned 254 patients presenting for routine echocardiography to Ain Shams University Hospitals Echocardiography unit; where clinical history was taken, examination and $2 \mathrm{D}$ echocardiography done where we indexed the elastic properties of the aorta by calculating of aortic distensibility (AD), aortic stiffness index (ASI), and pressure-strain elastic modulus (Ep), and LV diastolic function was evaluated as well.
\end{abstract}

Both presence and grade of left ventricular diastolic dysfunction were significantly correlated with each of arterial hypertension and diabetes mellitus separately and presence of both hypertension and diabetes mellitus was correlated with LVDD.

Aortic compliance-with its three studied elements; AS\%, AD and ASI-was significantly correlated with grade of LVDD yet only elastic modulus was independently correlated with diastolic dysfunction.

Keywords: Aortic stiffness; Diastolic dysfunction; Echocardiography; Arterial hypertension

\section{Introduction}

Arterial compliance and stiffness of the ascending aorta are important components of the elastic nature of the arterial system. Aortic elasticity plays an important role maintaining normal cardiovascular physiology due to the pulsatile flow generated by the cardiac pump [1]. Reduced aortic compliance and increased stiffness alter myocardial blood flow by decreasing diastolic perfusion pressure [1-6]. Several pathological conditions are known to potentially affect arterial compliance properties as occurs with arterial hypertension [2-4], and diabetes mellitus [5]. Aortic stiffness follow-up is important in several conditions as with follow up of Marfan syndrome and with bicuspid aortic valves and aortic aneurysms [7-10]. Left ventricular elasticity as represented by its diastolic function is increasingly recognized as an element of heart failure syndrome and is gaining attention as a separate entity requiring studies [11]. Factors known to affect LV elasticity are similar to those affecting aortic elasticity [12]. The aim of the current study is to further study relation between aortic stiffness and LV diastolic function and factors affecting each and how they are correlated.

\section{Patients and Methods}

Two hundred and fifty-four patients presenting to Ain Shams University Hospitals Echocardiography unit for routine checkup were scanned as follows:

Thorough clinical history including age, gender, diabetes mellitus, arterial hypertension, and prior cardiac history.

Physical examination to subjects was done recording height, body weight, BMI, ABP, and pulse rate. Before inclusion, we obtained an informed written consent from all patients after fully informing them of the study protocol, and the protocol was seen and approved by our local research ethical committee as it conforms to the 2002 revision of 1975 Declaration of Helsinki ethical guidelines. Echocardiography GE
(General Electric Company, Milwaukee, WI, USA) Vivid S5, 7 and E9 machines were used for echocardiographic evaluations where M4 and S3 probes transducer of $2.5 \mathrm{MHz}$ to $3.5 \mathrm{MHz}$ were utilized. All patients were evaluated with standard M-mode, two dimensional, Doppler, and tissue Doppler echocardiography views. Single-lead electrocardiography (ECG) recordings were made during the studies. Data was obtained by a single operator to avoid individual variation and was recorded for offline analysis. Measurements The left ventricular end-diastolic and end-systolic diameters and the left atrial end-systolic diameters were measured with $\mathrm{M}$-mode in the long axis parasternal view, following the guidelines of the American Society of Echocardiography [13]. Pulsed wave (PW) Doppler in apical four chamber view was utilized to assess mitral inflow velocities with the sample volume placed at the mitral leaflets tip. We initially classified Diastolic fillings depending on the early rapid filling wave "E" peak velocity, late filling wave "A" velocity, $\mathrm{E} / \mathrm{A}$ ratio, and deceleration time (DT), which is the time taken for the $\mathrm{E}$ wave to reach baseline. A $5 \mathrm{~mm}$ pulsed Doppler sample in the apical 4 chamber view was used for Tissue Doppler evaluation using as minimal optimal gain as possible in order to obtain best signal-to-noise ratio. We adjusted the spectral pulsed Doppler signal filter until Nyquist limit was $15 \mathrm{~cm} / \mathrm{s}$ to $20 \mathrm{~cm} / \mathrm{s}$ using a $3.5 \mathrm{MHz}$ to $4.0 \mathrm{MHz}$ frequency transducer. We set the sweep speed at $50 \mathrm{~mm} / \mathrm{s}$ in order to optimize myocardial velocities spectral display. We placed the PW doppler cursor on the lateral and septal mitral annulus of the left ventricle in the apical four-

${ }^{*}$ Corresponding authors: Adham Abdeltawab, Cardiology Department, Faculty of Medicine, Ain Shams University, Cairo, Egypt, Tel: 201008800788; Fax: 20224820416; E-mail: adham_abdeltawab@med.asu.edu.eg

\section{Received April 10, 2017; Accepted May 11, 2017; Published May 15, 2017}

Citation: Eweda I, Samir S, Abdeltawab A (2017) Relation Between LV Diastolic Function and Aortic Compliance as Assessed by Transthoracic Echo. J Cardiovasc Dis Diagn 5: 274. doi: 10.4172/2329-9517.1000274

Copyright: ( 2017 Eweda l, et al. This is an open-access article distributed under the terms of the Creative Commons Attribution License, which permits unrestricted use, distribution, and reproduction in any medium, provided the original author and source are credited. 
Citation: Eweda I, Samir S, Abdeltawab A (2017) Relation Between LV Diastolic Function and Aortic Compliance as Assessed by Transthoracic Echo. J Cardiovasc Dis Diagn 5: 274. doi: 10.4172/2329-9517.1000274

Page 2 of 5

chamber view, to obtain tissue Doppler spectral images. We obtained the myocardial peak early (Ea) and late diastolic (Aa) velocities from there. Aortic diameters were obtained from parasternal long axis view, at a level $3 \mathrm{~cm}$ above the aortic cusps. We obtained M-mode diameter measurements in systole (point of maximal anterior motion of aorta) and at end-diastole ( $Q$ wave on ECG).

We indexed the aortic elastic properties through calculating aortic stiffness index (ASI), aortic distensibility (AD), and pressure-strain elastic modulus (Ep), as follows:

$$
\begin{aligned}
& \mathrm{SI}=\ln (\mathrm{Ps} / \mathrm{Pd}) /([\text { As-Ad }] / \mathrm{Ad}), \\
& \mathrm{Ep}=(\mathrm{Ps}-\mathrm{Pd}) /([\text { As-Ad] } / \mathrm{Ad}), \text { and } \\
& \mathrm{AD}=2(\text { As-Ad) } /(\text { Ad }[\mathrm{Ps}-\mathrm{Pd}]),[3]
\end{aligned}
$$

where As is end systolic aortic diameter, Ad is end diastolic aortic diameter, $\mathrm{Ps}$ is systolic $\mathrm{ABP}, \mathrm{Pd}$ is diastolic $\mathrm{ABP}$, and $\ln$ is natural logarithm [14-16]. Statistical analyses we calculated continuous variables within group data as average \pm standard deviation, and categorical variables as numbers and percentages.

We used Kolmogorov-Smirnov statistics to check continuous variables for the normal distribution assumption. We used the Student's t-test to compare parametric variables and tested the categorical variables by Pearson's $\chi 2$ test and Fisher's exact test. We also used Binary logistic regression analysis to find possible independent association between Aortic stiffness and study parameters. We analyzed LV diastolic function parameters separately in multivariate regression analysis, in order to prevent multicollinearity. P-values are two sided, and values $<0.05$ were considered statistically significant. We used statistical package for social sciences (SPSS) software (v 20.0 for Windows; SPSS Inc., Chicago, IL, USA) to carry out all statistical analyses.

\section{Results}

\begin{tabular}{|c|c|c|c|c|}
\hline & Minimum & Maximum & Mean & Std. Deviation \\
\hline LVEDD (cm) & 3.30 & 6.50 & 4.84 & 0.54 \\
\hline LVESD (cm) & 2.00 & 4.70 & 3.09 & 0.42 \\
\hline PWT (cm) & 0.60 & 1.50 & 1.04 & 0.17 \\
\hline Sept $(\mathrm{cm})$ & 0.60 & 1.50 & 1.09 & 0.19 \\
\hline FS & 26.00 & 49.00 & 34.83 & 4.77 \\
\hline $\mathrm{EF}$ & 50.00 & 80.00 & 65.36 & 6.09 \\
\hline $\mathrm{LA}(\mathrm{cm})$ & 2.50 & 5.10 & 3.87 & 0.54 \\
\hline $\mathrm{AO} \operatorname{root}(\mathrm{cm})$ & 2.10 & 4.50 & 3.18 & 0.46 \\
\hline $\mathrm{AOS}(\mathrm{cm})$ & 2.50 & 4.60 & 3.42 & 0.45 \\
\hline $\mathrm{AOD}(\mathrm{cm})$ & 2.00 & 4.30 & 3.08 & 0.50 \\
\hline$E(\mathrm{~cm} / \mathrm{s})$ & 20.00 & 170.00 & 67.96 & 25.72 \\
\hline$A(\mathrm{~cm} / \mathrm{s})$ & 19.00 & 110.00 & 64.35 & 17.35 \\
\hline E/A ratio & 0.43 & 3.00 & 1.15 & 0.57 \\
\hline DT (msec) & 84.00 & 490.00 & 211.17 & 57.30 \\
\hline IVRT (msec) & 44.00 & 144.00 & 94.87 & 20.89 \\
\hline $\mathrm{Sa}(\mathrm{cm} / \mathrm{s})$ & 5.00 & 17.00 & 8.84 & 2.35 \\
\hline $\mathrm{Ea}(\mathrm{cm} / \mathrm{s})$ & 3.00 & 12.00 & 7.58 & 1.98 \\
\hline $\mathrm{Aa}(\mathrm{cm} / \mathrm{s})$ & 4.00 & 16.00 & 9.29 & 2.51 \\
\hline$E / E^{\prime}$ & 3.33 & 20.00 & 9.45 & 3.81 \\
\hline$E^{\prime} / A^{\prime}$ & 0.38 & 2.00 & 0.85 & 0.25 \\
\hline AS (\%) & 5.13 & 25.00 & 11.93 & 5.12 \\
\hline $\mathrm{AD}\left(\mathrm{cm}^{2} / \mathrm{dyn} / 10\right)$ & 0.15 & 1.28 & 0.49 & 0.28 \\
\hline ASI_MV & 0.02 & 0.10 & 0.05 & 0.02 \\
\hline
\end{tabular}

We enrolled a total of 254 subjects in the study, with $50.4 \%$ females, mean age 56 years \pm 10 , with tendency to obesity; mean BMI $31.6 \pm$

\begin{tabular}{|c|c|c|c|c|c|c|c|c|c|c|}
\hline \multirow{3}{*}{$\begin{array}{l}\text { DDLV } \\
\text { Grade }\end{array}$} & \multicolumn{8}{|c|}{ Disease Status } & \multirow{3}{*}{$\begin{array}{l}\text { Chi- } \\
\text { square }\end{array}$} & \multirow{3}{*}{$\begin{array}{c}\text { P- } \\
\text { value }\end{array}$} \\
\hline & \multicolumn{2}{|c|}{ Negative } & \multicolumn{2}{|c|}{ Hypertension } & \multicolumn{2}{|c|}{ Diabetes } & \multicolumn{2}{|c|}{ Both } & & \\
\hline & No. & $\%$ & No. & $\%$ & No & $\%$ & No. & $\%$ & & \\
\hline I & 35 & $41.20 \%$ & 16 & $17.60 \%$ & 6 & $28.60 \%$ & 4 & $7.00 \%$ & \multirow{3}{*}{139.34} & \multirow{3}{*}{$0.000^{* *}$} \\
\hline II & 50 & $58.80 \%$ & 73 & $80.20 \%$ & 15 & $71.40 \%$ & 19 & $33.30 \%$ & & \\
\hline III & 0 & $0.00 \%$ & 2 & $2.20 \%$ & 0 & $0.00 \%$ & 34 & $59.60 \%$ & & \\
\hline
\end{tabular}

Table 1: Descriptive statistics of echo parameters studied.

\begin{tabular}{|c|c|c|c|c|c|c|c|}
\hline \multicolumn{2}{|c|}{$\begin{array}{l}\text { LV Functions } \\
\text { Parameters }\end{array}$} & Mean & $\begin{array}{c}\text { Std. } \\
\text { Deviation }\end{array}$ & Minimum & Maximum & ANOVA & P-value \\
\hline \multirow{3}{*}{ AS (\%) } & 1 & 14.81 & 4.37 & 6.06 & 25.00 & \multirow{3}{*}{49.68} & \multirow{3}{*}{$0.000^{* *}$} \\
\hline & II & 12.22 & 4.80 & 5.26 & 23.81 & & \\
\hline & III & 5.79 & 0.36 & 5.13 & 6.98 & & \\
\hline \multirow{3}{*}{$\begin{array}{l}\mathrm{AD}\left(\mathrm{cm}^{2} /\right. \\
\left.\text { dyn } / 10^{-1}\right)\end{array}$} & I & 0.65 & 0.25 & 0.24 & 1.23 & \multirow{3}{*}{42.94} & \multirow{3}{*}{$0.000^{* *}$} \\
\hline & II & 0.51 & 0.27 & 0.19 & 1.28 & & \\
\hline & III & 0.17 & 0.01 & 0.15 & 0.20 & & \\
\hline \multirow{3}{*}{ ASI_MV } & I & 0.03 & 0.01 & 0.02 & 0.07 & \multirow{3}{*}{225.30} & \multirow{3}{*}{$0.000^{* *}$} \\
\hline & II & 0.04 & 0.02 & 0.02 & 0.08 & & \\
\hline & III & 0.09 & 0.00 & 0.08 & 0.10 & & \\
\hline Hiable & & Sign & ificant at $P$ & 0.01 & & & \\
\hline
\end{tabular}

$\left({ }^{* *}\right)$ Highly Statistically Significant at $\mathrm{P}<0.01$

Table 2: Relation between disease status and LVDD Grade.

Table 3: Correlation between LV diastolic function and aortic compliance.

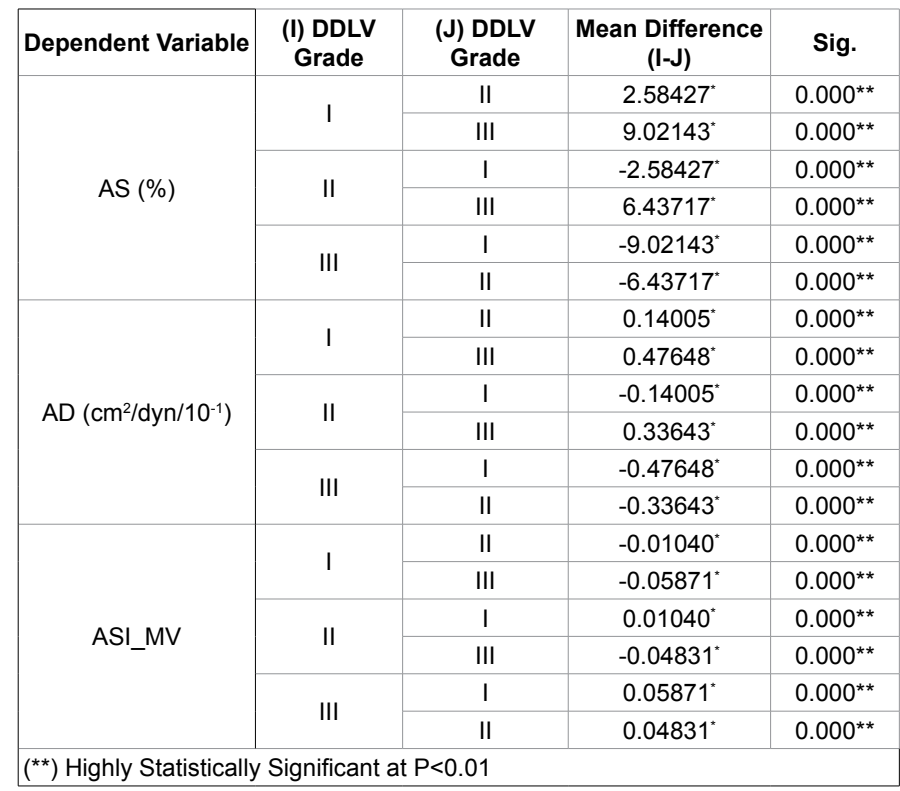

Table 4: Post-hoc test.

5.16, mean ABP 140/87 mmHg. Of the included patients 58.3\% were hypertensive and $30.7 \%$ were diabetic. Of the studied subjects, $24 \%$ had LVDD grade I, $61.8 \%$ grade II and $14.2 \%$ grade III.

As regards the echo parameters; mean LVEF was $65.4 \% \pm 6$, AS\% mean $11.9 \pm 5.1$, mean $\mathrm{AD}$ was $0.49 \pm 0.28$, and aortic stiffness index mean $0.0484 \pm 0.023$. full descriptive analysis for echo parameters shown in Table 1.

Presence of LVDD and its grade was significantly correlated with each of HTN and DM and presence of both together was correlated with LVDD with high statistical significance (Table 2).

Aortic compliance was correlated with grade of LVDD with high statistical significance with its three studied elements; AS\%, AD and ASI (Tables 3 and 4). 
Aortic stiffness was significantly correlated with disease status (DM, and HTN), ABP (both systolic and diastolic), aortic root diameter, septal thickness, A wave velocity, and E/A ratio, yet it was not found to be significantly correlated with degree of LVDD (Table 5).

Aortic distensibility was also significantly correlated with disease status (DM, and HTN), ABP (both systolic and diastolic), aortic root diameter, septal thickness, A wave velocity, and Sa wave velocity by TDI, yet it was also not found to be significantly correlated with degree of LVDD (Table 6).

Disease status (DM, and HTN), ABP (both systolic and diastolic), aortic root diameter, LVEF, LA diameter, E wave velocity, and IVRT were significantly correlated with ASI-MV. ASI-MV was significantly correlated to the degree of LVDD (Table 7).

\section{Discussion}

The current study was a prospective observational study aiming at studying relation between aortic stiffness and LV diastolic function

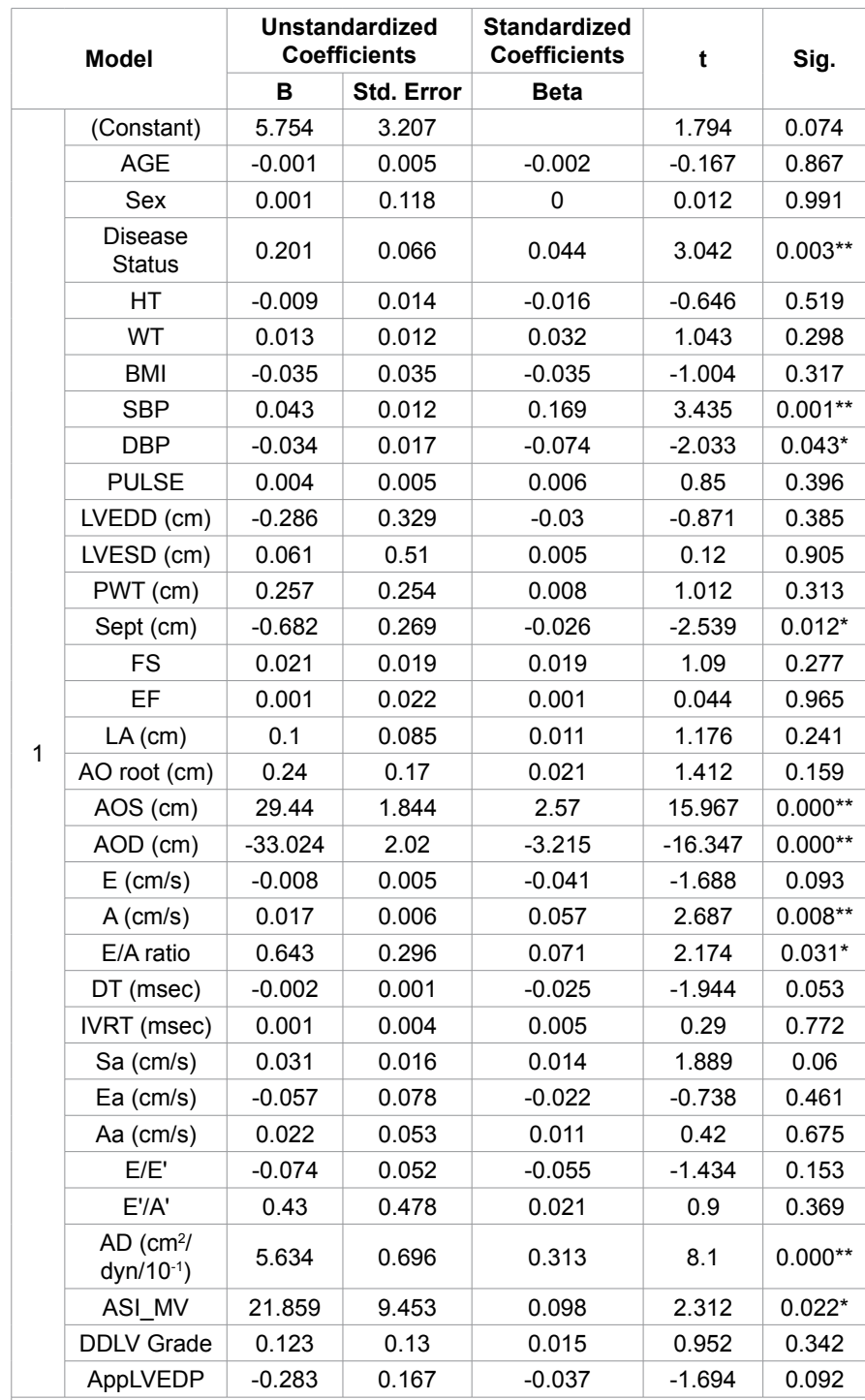

a. Dependent Variable: AS $(\%) ;\left({ }^{* \star}\right)$ Highly Statistically Significant at $P<0.01 ;\left(^{*}\right)$ Statistically Significant at $\mathrm{P}<0.05$

Table 5: Multiple linear regression showing independent predictors of AS coefficients $^{\mathrm{a}}$.

\begin{tabular}{|c|c|c|c|c|c|c|}
\hline \multirow{2}{*}{\multicolumn{2}{|c|}{ Model }} & \multicolumn{2}{|c|}{$\begin{array}{l}\text { Unstandardized } \\
\text { Coefficients }\end{array}$} & \multirow{2}{*}{\begin{tabular}{|c|}
$\begin{array}{c}\text { Standardized } \\
\text { Coefficients }\end{array}$ \\
Beta
\end{tabular}} & \multirow[t]{2}{*}{$\mathbf{t}$} & \multirow[t]{2}{*}{ Sig. } \\
\hline & & B & Std. Error & & & \\
\hline \multirow{34}{*}{1} & (Constant) & 0.487 & 0.273 & & 1.784 & 0.076 \\
\hline & Age & 0 & 0 & -0.008 & -0.565 & 0.573 \\
\hline & Sex & -0.008 & 0.01 & -0.014 & -0.815 & 0.416 \\
\hline & $\begin{array}{l}\text { Disease } \\
\text { Status }\end{array}$ & -0.019 & 0.006 & -0.074 & -3.331 & $0.001^{* *}$ \\
\hline & $\mathrm{HT}$ & -0.001 & 0.001 & -0.028 & -0.729 & 0.467 \\
\hline & WT & 0.001 & 0.001 & 0.042 & 0.894 & 0.372 \\
\hline & BMI & -0.003 & 0.003 & -0.047 & -0.869 & 0.386 \\
\hline & SBP & -0.013 & 0.001 & -0.907 & -19.119 & $0.000^{* *}$ \\
\hline & DBP & 0.015 & 0.001 & 0.575 & 14.261 & $0.000^{* *}$ \\
\hline & PULSE & 0 & 0 & 0.009 & 0.799 & 0.425 \\
\hline & LVEDD (cm) & 0.003 & 0.028 & 0.005 & 0.096 & 0.923 \\
\hline & LVESD (cm) & 0.005 & 0.043 & 0.008 & 0.124 & 0.901 \\
\hline & PWT (cm) & -0.007 & 0.022 & -0.004 & -0.326 & 0.744 \\
\hline & Sept (cm) & 0.025 & 0.023 & 0.017 & 1.097 & 0.274 \\
\hline & $\mathrm{FS}$ & 0.004 & 0.002 & 0.071 & 2.655 & $0.009^{* *}$ \\
\hline & $\mathrm{EF}$ & -0.003 & 0.002 & -0.059 & -1.448 & 0.149 \\
\hline & $\mathrm{LA}(\mathrm{cm})$ & -0.014 & 0.007 & -0.026 & -1.887 & 0.06 \\
\hline & AO root $(\mathrm{cm})$ & -0.021 & 0.014 & -0.033 & -1.435 & 0.153 \\
\hline & $\mathrm{AOS}(\mathrm{cm})$ & 0.536 & 0.228 & 0.841 & 2.356 & $0.019^{*}$ \\
\hline & $\mathrm{AOD}(\mathrm{cm})$ & -0.553 & 0.253 & -0.967 & -2.184 & $0.030^{*}$ \\
\hline & $\mathrm{E}(\mathrm{cm} / \mathrm{s})$ & 0.001 & 0 & 0.06 & 1.62 & 0.107 \\
\hline & $\mathrm{A}(\mathrm{cm} / \mathrm{s})$ & -0.001 & 0.001 & -0.085 & -2.595 & $0.010^{*}$ \\
\hline & E/A ratio & -0.041 & 0.025 & -0.083 & -1.637 & 0.103 \\
\hline & DT (msec) & $\begin{array}{l}6.28 \mathrm{E}- \\
05\end{array}$ & 0 & 0.013 & 0.64 & 0.523 \\
\hline & IVRT (msec) & 0 & 0 & -0.021 & -0.802 & 0.423 \\
\hline & $\mathrm{Sa}(\mathrm{cm} / \mathrm{s})$ & -0.003 & 0.001 & -0.027 & -2.348 & $0.020^{*}$ \\
\hline & $\mathrm{Ea}(\mathrm{cm} / \mathrm{s})$ & 0.009 & 0.007 & 0.064 & 1.405 & 0.162 \\
\hline & $\mathrm{Aa}(\mathrm{cm} / \mathrm{s})$ & 0.002 & 0.004 & 0.015 & 0.37 & 0.712 \\
\hline & $E / E^{\prime}$ & 0.008 & 0.004 & 0.105 & 1.792 & 0.075 \\
\hline & $E^{\prime} / A^{\prime}$ & -0.009 & 0.041 & -0.008 & -0.215 & 0.83 \\
\hline & ASI_MV & 4.001 & 0.768 & 0.323 & 5.21 & $0.000^{* *}$ \\
\hline & DDLV_Grade & -0.014 & 0.011 & -0.03 & -1.265 & 0.207 \\
\hline & AppLVEDP_c & 0.007 & 0.014 & 0.016 & 0.487 & 0.627 \\
\hline & AS (\%) & 0.041 & 0.005 & 0.733 & 8.1 & $0.000^{* *}$ \\
\hline
\end{tabular}

a Dependent Variable: $A D\left(\mathrm{~cm}^{2} / \mathrm{dyn} / 10^{-1}\right) ;\left({ }^{* *}\right)$ Highly Statistically Significant at $\mathrm{P}<0.01\left(^{*}\right)$ Statistically Significant at $\mathrm{P}<0.05$

Table 6: Multiple linear regression showing independent predictors of $A D$

and factors affecting each and how they are correlated. It included 254 patients, with almost equal gender distribution, mean age 56 years \pm 10 , with tendency to obesity. Of the studied subjects, 24\% had LVDD grade I, $61.8 \%$ grade II and $14.2 \%$ grade III. Presence of LVDD and its grade was significantly correlated with each of HTN and DM and presence of both together was correlated with LVDD. Aortic compliance-with its three studied elements; AS\%, AD and ASI-was significantly correlated with grade of LVDD. Aortic stiffness and distensibility were found to be significantly correlated with disease status, ABP, septal thickness, A wave velocity, and aortic root diameter, yet neither was found to be significantly correlated with degree of LVDD. ASI-MV was also found to be significantly correlated to disease status, ABP, aortic root diameter, LVEF, LA diameter, E wave velocity, IVRT, and degree of LVDD. Eren, et al. [17] studied relation of aortic stiffness to LV diastolic function in patients without coronary artery disease, with hypertension, diabetes or both. Study groups were composed of 27 healthy participants and 25 patients with hypertension, 24 with diabetes, and 18 with hypertension and diabetes. Their conclusion was that aortic stiffness increased with hypertension, diabetes, or both even after excluding coronary artery 


\begin{tabular}{|c|c|c|c|c|c|}
\hline \multirow[t]{2}{*}{ Model } & \multicolumn{2}{|c|}{$\begin{array}{l}\text { Unstandardized } \\
\text { Coefficients }\end{array}$} & \multirow{2}{*}{$\begin{array}{c}\text { Standardized } \\
\text { Coefficients } \\
\text { Beta }\end{array}$} & \multirow[t]{2}{*}{$\mathbf{t}$} & \multirow[t]{2}{*}{ Sig. } \\
\hline & B & Std. Error & & & \\
\hline (Constant) & -0.065 & 0.022 & & -2.93 & 0.004 \\
\hline Age & 2.10E-05 & 0 & 0.009 & 0.646 & 0.519 \\
\hline Sex & -0.001 & 0.001 & -0.022 & -1.247 & 0.214 \\
\hline $\begin{array}{l}\text { Disease } \\
\text { Status }\end{array}$ & 0.003 & 0 & 0.128 & 5.875 & $0.000^{* *}$ \\
\hline $\mathrm{HT}$ & 0 & 0 & 0.087 & 2.229 & $0.027^{*}$ \\
\hline WT & 0 & 0 & -0.119 & -2.487 & $0.014^{*}$ \\
\hline BMI & 0.001 & 0 & 0.139 & 2.568 & $0.011^{*}$ \\
\hline SBP & 0.001 & 0 & 0.731 & 11.746 & $0.000^{* *}$ \\
\hline DBP & -0.001 & 0 & -0.567 & -13.233 & $0.000^{* *}$ \\
\hline PULSE & $-5.66 \mathrm{E}-05$ & 0 & -0.018 & -1.685 & 0.093 \\
\hline LVEDD (cm) & -0.003 & 0.002 & -0.063 & -1.153 & 0.25 \\
\hline LVESD (cm) & 0.005 & 0.004 & 0.089 & 1.366 & 0.173 \\
\hline PWT (cm) & 0 & 0.002 & -0.003 & -0.231 & 0.818 \\
\hline Sept (cm) & 0.001 & 0.002 & 0.007 & 0.421 & 0.674 \\
\hline FS & 0 & 0 & -0.084 & -3.081 & $0.002^{* *}$ \\
\hline EF & 0 & 0 & 0.1 & 2.44 & $0.015^{*}$ \\
\hline LA (cm) & 0.002 & 0.001 & 0.046 & 3.369 & $0.001^{* *}$ \\
\hline AO root $(\mathrm{cm})$ & -0.002 & 0.001 & -0.043 & -1.82 & 0.07 \\
\hline $\mathrm{AOS}(\mathrm{cm})$ & -0.173 & 0.015 & -3.355 & -11.41 & $0.000^{* *}$ \\
\hline $\mathrm{AOD}(\mathrm{cm})$ & 0.193 & 0.017 & 4.172 & 11.501 & $0.000^{* *}$ \\
\hline $\mathrm{E}(\mathrm{cm} / \mathrm{s})$ & $-8.72 \mathrm{E}-05$ & 0 & -0.098 & -2.586 & $0.010^{*}$ \\
\hline $\mathrm{A}(\mathrm{cm} / \mathrm{s})$ & 4.97E-05 & 0 & 0.038 & 1.106 & 0.27 \\
\hline E/A ratio & 0.005 & 0.002 & 0.123 & 2.396 & 0.017 \\
\hline DT (msec) & $1.28 \mathrm{E}-05$ & 0 & 0.032 & 1.589 & 0.114 \\
\hline IVRT (msec) & $-6.86 \mathrm{E}-05$ & 0 & -0.062 & -2.372 & $0.019^{*}$ \\
\hline $\mathrm{Sa}(\mathrm{cm} / \mathrm{s})$ & $9.41 \mathrm{E}-07$ & 0 & 0 & 0.008 & 0.994 \\
\hline $\mathrm{Ea}(\mathrm{cm} / \mathrm{s})$ & 0 & 0.001 & 0.01 & 0.216 & 0.829 \\
\hline $\mathrm{Aa}(\mathrm{cm} / \mathrm{s})$ & 0 & 0 & 0.051 & 1.252 & 0.212 \\
\hline E/E' & 0.001 & 0 & 0.094 & 1.553 & 0.122 \\
\hline$E^{\prime} / A^{\prime}$ & 0.004 & 0.003 & 0.04 & 1.109 & 0.269 \\
\hline DDLV Grade & 0.002 & 0.001 & 0.059 & 2.449 & $0.015^{*}$ \\
\hline AppLVEDP c & 0.001 & 0.001 & 0.016 & 0.457 & 0.648 \\
\hline AS (\%) & 0.001 & 0 & 0.242 & 2.312 & $0.022^{*}$ \\
\hline $\begin{array}{l}\mathrm{AD}\left(\mathrm{cm}^{2} /\right. \\
\text { dyn/10-1) }\end{array}$ & 0.027 & 0.005 & 0.34 & 5.21 & $0.000^{\star *}$ \\
\hline
\end{tabular}

a. Dependent Variable: ASI_MV; $\left(^{* *}\right)$ Highly Statistically Significant at $P<0.01\left(^{*}\right)$ Statistically Significant at $\mathrm{P}<0.05$

Table 7: Multiple linear regression showing independent predictors of AS coefficients ${ }^{\mathrm{a}}$.

disease. They also found aortic stiffness and left ventricular diastolic dysfunction to be associated. Cesare Russo et al. [18] studied gender difference in arterial stiffness and its relation to diastolic function, and found general arterial stiffness to be correlated with female gender which reflected the higher incidence of diastolic dysfunction in females. Similar results correlating general arterial stiffness-as reflected by pulse wave velocity, central pulse pressure/stroke volume index, total arterial compliance, pulse pressure amplification, and augmentation indexwith diastolic function was found by Zito et al. [19], Agoşton-Coldea et al. [20], and Mottram et al. [21].

Kasikcioglu et al. [22] studied left ventricular diastolic function and aortic distensibility in thirty male runners and thirty age-matched healthy male controls and found better diastolic function in endurance athletes that reflected the increased aortic distensibility found. Kristin et al. [23] retrospectively compared echocardiograms of 24 children with repair of isolated coacrtation of the aorta and 24 matched controls in order to determine the relation between aortic stiffness and left ventricular diastolic dysfunction and found that even children who

underwent CoA repair early have abnormal LV diastolic function and aortic elasticity compared with controls and found a linear relation between the two.

Ikonomidis et al. [24] studied LV diastolic function and aortic elastic properties in patients with Behcet's disease and concluded that aortic elastic properties and left ventricular diastolic function are both impaired in patients with Behcet's disease and are interrelated, and suggested the presence of a common pathophysiologic pathway which provided a possible marker of risk for vascular disease.

\section{Conclusion}

Presence of LVDD and its grade was significantly correlated with each of HTN and DM and presence of both together was correlated with LVDD. Aortic compliance-with its three studied elements; AS\%, AD and ASI-was found to be significantly correlated with grade of LVDD, yet neither AS\% nor AD was independently significantly correlated with LVDD grade. Only ASI-MV was significantly correlated degree of LVDD independent of other factors. Relation of aortic stiffness to diastolic function may be largely attributed to common causative factors.

\section{References}

1. Elzinga G, Westerhof N (1973) Pressure and flow generated by the left ventricle against different impedances. Circ Res 32: 178-186.

2. Peralta CA, Adeney KL, Shlipak MG, Jacobs D Jr, Duprez D, et al. (2010) Structural and functional vascular alterations and incident hypertension in normotensive adults: The multi-ethnic study of atherosclerosis. Am J Epidemio 171: 63-71.

3. Redheuil A, Yu WC, Wu CO, Mousseaux E, de Cesare A, et al. (2010) Reduced ascending aortic strain and distensibility: Earliest manifestations of vascular aging in humans. Hypertension 55: 319-326

4. Vitarelli A, Giordano M, Germano G, Pergolini M, Cicconetti P, et al. (2010) Assessment of ascending aorta wall stiffness in hypertensive patients by tissue Doppler imaging and strain Doppler echocardiography. Heart 96: 1469-1474.

5. Stakos DA, Schuster DP, Sparks EA, Wooley CF, Osei K, et al. (2005) Cardiovascular effects of type 1 diabetes mellitus in children. Angiology 56 311-317.

6. Murgo JP, Westerhof N, Giolma JP, Altobelli SA (1980) Aortic input impedance in normal man: Relationship to pressure wave forms. Circulation 62: 105-116.

7. Baumgartner D, Baumgartner C, Schermer E, Engl G, Schweigmann U, et al. (2006) Different patterns of aortic wall elasticity in patients with Marfan syndrome: A noninvasive follow-up study. J Thorac Cardiovasc Surg 132: 811-819.

8. Grotenhuis HB, Ottenkamp J, Westenberg JJ, Bax JJ, Kroft LJ, et al. (2007) Reduced aortic elasticity and dilatation are associated with aortic regurgitation and left ventricular hypertrophy in non-stenotic bicuspid aortic valve patients. $J$ Am Coll Cardiol 49: 1660-1665.

9. Nistri S, Sorbo MD, Basso C, Thiene G (2002) Bicuspid aortic valve: Abnorma aortic elastic properties. The J heart valve Dis11: 369-373.

10. Pober BR, Johnson M, Urban Z (2008) Mechanisms and treatment of cardiovascular disease in Williams-Beuren syndrome. J Clin Invest 118 1606-1615.

11. EIGuindy A, Yacoub MH (2012) Heart failure with preserved ejection fraction Global Cardiol Sci Prac 2012: 10.

12. Bia D, Aguirre I, Zócalo Y, Devera L, Cabrera Fischer E, et al. (2005) Regional differences in viscosity, elasticity and wall buffering function in systemic arteries: Pulse wave analysis of the arterial pressure-diameter relationship. Spanish J Cardiol 58: 167-174.

13. Lang RM, Bierig M, Devereux RB, Flachskampf FA, Foster E, et al. (2005) Recommendations for chamber quantification: A report from the American Society of Echocardiography's Guidelines and Standards Committee and the Chamber Quantification Writing Group, developed in conjunction with the European Association of Echocardiography, a branch of the European Society of Cardiology. J Am Soc Echocardiogr 18: 1440-1463. 
Citation: Eweda I, Samir S, Abdeltawab A (2017) Relation Between LV Diastolic Function and Aortic Compliance as Assessed by Transthoracic Echo. J Cardiovasc Dis Diagn 5: 274. doi: 10.4172/2329-9517.1000274

Page 5 of 5

14. Dernellis J, Panaretou M (2005) Aortic stiffness is an independent predictor of progression to hypertension in nonhypertensive subjects. Hypertension 45 : 426-431.

15. Nemes A, Geleijnse ML, Forster T, Soliman OI, Ten Cate FJ, et al. (2008) Echocardiographic evaluation and clinical implications of aortic stiffness and coronary flow reserve and their relation. Clin Cardiol 31: 304-309.

16. Stefanadis C, Stratos C, Boudoulas H, Kourouklis C, Toutouzas P (1990) Distensibility of the ascending aorta: Comparison of invasive and non-invasive techniques in healthy men and in men with coronary artery disease. Eur Heart J 11: 990-996.

17. Eren M, Gorgulu S, Uslu N, Celik S, Dagdeviren B, et al. (2004) Relation between aortic stiffness and left ventricular diastolic function in patients with hypertension, diabetes, or both. Heart 90: 37-43.

18. Russo C, Jin Z, Palmieri V, Homma S, Rundek T, et al. (2012) Arterial stiffness and wave reflection: Sex differences and relationship with left ventricular diastolic function. Hypertension 60: 362-368.

19. Zito C, Mohammed M, Todaro MC, Khandheria BK, Cusma-Piccione M, et al.
(2014) Interplay between arterial stiffness and diastolic function: A marker of ventricular-vascular coupling. J Cardiovasc Med (Hagerstown) 15: 788-796.

20. Agoston-Coldea L, Mocan T, C. Bobar, (2008) Arterial stiffness and left ventricular diastolic function in the patients with hypertension. Rom $\mathrm{J}$ Intern Med 46: 313-321.

21. Mottram PM, Haluska BA, Leano R, Carlier S, Case C, et al. (2005) Relation of arterial stiffness to diastolic dysfunction in hypertensive heart disease. Heart 91: 1551-1556.

22. Kasikcioglu E, Kayserilioglu A, Oflaz H, Akhan H (2005) Aortic distensibility and left ventricular diastolic functions in endurance athletes. Int J Sports Med 26: $165-170$.

23. Lombardi KC, Northrup V, McNamara RL, Sugeng L, Weismann CG (2013) Aortic stiffness and left ventricular diastolic function in children following early repair of aortic coarctation. Am J Cardiol 112: 1828-1833.

24. Ikonomidis I, Lekakis J, Stamatelopoulos K, Markomihelakis N, Kaklamanis PG, et al. (2004) Aortic elastic properties and left ventricular diastolic function in patients with Adamantiades-Behcet's disease. J Am Coll Cardiol 43: 1075-1081. 